\title{
A personal note-Gerald Stern and Yves Agid: A walk in Piazza del Popolo in Rome, sometime in 1998
}

\author{
Yves Agid $^{1}$
}

Published online: 24 March 2020

C) Springer-Verlag GmbH Austria, part of Springer Nature 2020

Gerald: "Do you remember that as recently as a few years ago, care for nervous system diseases was considered primarily a contemplative activity? Fortunately, it's no longer the case. Is it not remarkable that so much progress has been made recently? No one would have predicted it. Do not you think that it's largely due to the fact that medicine is now firmly anchored in an ongoing scientific base?

Yves: "I certainly think so. But we are still at the dawn of the medical middle ages Take the example of our discipline, we are only beginning to understand how the brain functions and what happens when it dysfunctions. Nevertheless, our daily practice has been markedly changed over the past years. Now, as an educator, how would you see the future delivery of medical care on a day to day basis"?

G: "In neurology and psychiatry, there are so many upheavals that will modify the management of patients. I am referring to the 4"Ps" that everyone have in mind: medicine that will become predictive, personalized, preventive, and participative"!

Y: "Is not your description too simplistic? If I respectively refer to each of your"P"s, do you really think that we will 'take care of patients without symptoms,' that we will be moving towards the concept of 'one patient, one symptom,' that we will be 'treating patients without symptoms,' that we will practice a sort of 'medicine without doctors'?

G: "Really, I do not see how we can escape it. However, as the discipline of neurology is essentially behaviorally based, I believe more in the quality of our semiology and our listening".

Y: "What you say is particularly true in the field of neuropsychology and movement disorders, where the clinical features in patients are so subtle and complex. But the possibility to use big data and artificial intelligence will soon completely modify the way we are taking care of patients,

Yves Agid

yves.agid@icm-institute.org

1 Paris, France do not you agree? What will it mean to be a good movement disorder doctor in 2020 ? He, or she, will probably have to become more of a good informatician, and less a good semiologist?

G: "What you say might indeed be true for the rough diagnosis of symptoms and syndromes. Then the question remains: to what extent will these new technologies help the practitioners in their daily care of patients suffering from neurological disorders?

Y: "Are you excluding psychiatry"?

G: "Certainly not, since the methods available for diagnosing and treating neurological and psychiatric patients are more or less the same, even if the clinical management of patients is clearly different."

Y: "By the way, this points to an integration of the two disciplines. Everyone knows that disabled neurological patients can suffer from psychiatric symptoms; and conversely, that most psychiatric disorders have an organic basis. After all, both disciplines are dealing with the brain, and hence the mind!"

G: "I agree. This is also true for the treatments of patients. We know how to comfort patients in both disciplines, using efficacious drugs such as antidepressants, neuroleptics, anxiolytics, antalgics, levodopa, antiepileptics, etc. “

Y: "Efficient drugs, yes—but up to a certain extent!"

G: "That's right. Moreover, these symptomatic compounds have been discovered a long time ago. But let me approach the most important question: What about the future of curative treatments? None is available presently. How are we going to stop a given pathological process? Despite the enormous progress of neurosciences in recent years, we are still unable to cure disorders such as Alzheimer, Parkinson, and other neurodegenerative disorders, as well as multiple sclerosis, epilepsy, schizophrenia, autism, etc. Why such a delay in terms of curative or predictive treatment compared to other disciplines such as oncology, for instance?

Y: "You are right, compared to other disciplines, we are still late to identify new curative treatments! Nevertheless, 
we have made impressive scientific progresses in the recent years! Please allow me to offer some not-so-trivial examples:

We have identified mutations of numerous hereditary diseases, taking advantage of the fantastic advances in the field of neurogenetics.

New neural brain imaging techniques have transformed our practice by enabling the localization and often the identification of most lesions of the nervous system.

We are beginning to understand the function and dysfunction of major neuronal pathways in an increasing number of pathologies using the latest neurophysiological technics.

Neuronal modelling and systems analysis allow us now to interpret an increasing number of health-related data, and to induce the rapid development of digital medicine.

Aren't all these advances outstanding"?

G: "Yes, of course, but why are we still unable to stop the evolution of any kind of neurodegenerative disorder? When will we be able to eliminate one for the first time, or even to delay the onset of a rare form of neurodegeneration"?

Y: "The reason for these unsuccessful attempts resides in one word: "complexity." The brain possesses a complexity that defies the imagination, at contrast with other organs. As you know much better than I do, the physiology of the nervous system is far more complex than that of a prostate or a liver. The human brain that has produced Shakespeare, Descartes, or Einstein is nothing but a sort of small-scale version of the complexity of the universe! We are just starting to identify the main neuronal circuits in the brain and their cellular interactions, but we still do not know how this material brain can produce an "immaterial" thought! We start to decipher the cell biology of neurons and glial cells, but, so far, despite thousands of trials, we have not been able to find a single pharmacological target capable of reducing the dysfunction of a neurodegenerative process".

G: "Before finding a treatment to cure a neurodegenerative disorder, do not you think we should first try to understand the nature of the elementary mechanisms that are at the origin of the brain's molecular and cellular processing? How are neurons, known to produce action potentials thereby eliciting the release of neurotransmitters, able to produce thought, a "non-substance" which is colorless, odorless, tasteless? What is the neural code? We are able to understand how the brain receives information from the environment, and how it produces actions modifying the environment in turn, but the way this information is processed within the brain is still unknown."

Y: "If you assume that a thought is information, then it's like a computer"?

G: "But it's not! My computer does not think. It has no emotions, no consciousness. It is unable to produce abstract reasoning. It is stupid"!

Y: "Yes, but your computer has incredible memory capacities. Like the best computers available so far, our brain is able to process myriads of information. The difference is that information processed in our computers is meaningless; whereas, information processed in our brain does mean something. If we assume that our thoughts consist in information, then, I do not see why we should not soon discover the brain's neural code. Most informaticians state that neuronal information acquires a meaning simply by increasing the complexity of the software of the brain, in other words, to increase the speed and the dynamics of neuronal interactions.

G: "If you consider that the brain is a machine, as I know you do believe, do you think that this machine can act, can perceive, and can think autonomously"?

Y: "I do not see any other possibility".

G: "I can see that you are not a dualist in the Cartesian sense; although you Frenchmen are supposed to be Cartesian in your analysis.

Y: "Most neuroscientists believe that the secret of thought's production resides in the complex, still enigmatic, cybernetics of neuronal circuits organized in various interrelated hubs. If I were to bet, I would include not only neurons but also other compounds of the nervous system, namely glial cells, capillaries which are in relation with the rest of our organism".

G:" Shall we wait until we understand the neural code to use the available scientific knowledge to discover new ways to cure intractable diseases of the nervous system"?

Y: "The problem today is not to discuss whether or not we should use our intelligence to find new pharmacological or surgical targets, this is mandatory in medicine. Today, our real challenge is to anticipate the type of medicine that will be implemented in the next few years. I am referring to the rapid development of telemedicine, digital medicine, brain machine interface, and other informatics-related devices that will soon transform our medical praxis. How do you see the future of medicine in general, and neurology in particular"?

G: "More than ever, of primary importance is the training of young neurologists, psychiatrists, and neurosurgeons. Whether a medical student wishes to specialize in a given subspecialty, he or she needs to complete appropriate scientific training.

Y: Yes, this is true, to anticipate the type of medicine they will practice 10 years later. This means we have to train teams of both "doctor-researchers" who will carry out research at the bedside, and "researcher-doctors" who will work at the bench. Different from pure clinicians and pure scientists, who have difficulties in comprehending each other, these two categories of investigators will then be able to understand each other's approaches. These two categories of doctors will, in turn, be able to forward this medicoscientific knowledge to the pure clinicians and researchers, respectively. This is mandatory to create bridges between clinical and research practices. 
Both: "... for the even greater benefit of patients."

G: "To become a good medical doctor in the future will not be easy! If I follow your proposal, good movement disorder doctors will need to be not only good medical internists and good movement disorder specialists, but, in addition, they will need also to be good scientists! If you are right, I am afraid that no one will want to become a student in the field of movement disorders!

Y: "Oh yes... they will".

G: "Why don't we have a glass of Brunello...!"

Y: "I was thinking the same"
G: "Yes."

Y: "A ta santé."

G: "Cheers."

Publisher's Note Springer Nature remains neutral with regard to jurisdictional claims in published maps and institutional affiliations. 